\title{
Successful treatment of post-intubation tracheal stenosis with balloon dilation, argon plasma coagulation, electrocautery and application of mitomycin C
}

\author{
Audra Fuller MD, Mark Sigler MD, Shrinivas Kambali MD, Raed Alalawi MD
}

\begin{abstract}
Tracheal stenosis is an uncommon but known complication of endotracheal intubation and tracheostomy. Surgery is currently the definitive treatment for tracheal stenosis but carries a significantly higher risk for adverse events as it often involves complex procedures. Here we present our experience using topical application of mitomycin $C$, along with various bronchoscopic interventions, as a treatment for tracheal stenosis. The patients in our series developed tracheal stenosis post-intubation or post-tracheostomy. Each patient in our series underwent the same basic procedures involving rigid bronchoscopy and balloon dilation, ablation of granulation tissue with cryotherapy and argon plasma coagulation, and finally application of topical mitomycin C. Our experience with these four cases shows a good initial success rate with topical mitomycin $C$ application for the treatment of tracheal stenosis. Most patients required a repeat intervention a few weeks later. Our results suggest that success with topical mitomycin $C$ is more likely in post-intubation rather than post-tracheostomy tracheal stenosis. Bronchoscopic therapy and topical application of mitomycin $\mathrm{C}$ may work better as a bridge to definitive surgery rather than as a stand-alone therapy.
\end{abstract}

Key words: Tracheal stenosis, bronchoscopy, topical mitomycin C, post-intubation, post-tracheostomy, airway obstruction

Corresponding author: Raed Alalawi

Contact Information: raed.alalawi@ttuhsc.edu

DOI: 10.12746/swrccc2015.0309.113 


\section{INTRODUCTION}

Tracheal stenosis is an uncommon but known complication of endotracheal intubation and tracheostomy. Here we present our experience with balloon dilation, argon plasma coagulation (APC), endoscopic electrosurgery (EES), and topical application of mitomycin $\mathrm{C}$ for the treatment for tracheal stenosis.

\section{Methods}

All patients in our series underwent the same basic procedures as described here in the bronchoscopy suite at University Medical Center in Lubbock, TX. First, each patient underwent rigid bronchoscopy and balloon dilation. Then the patient's granulation tissue was ablated with cryotherapy and APC. After these procedures the stenotic areas was treated with topical mitomycin $\mathrm{C}$. Small pieces of gauze were soaked with the mitomycin $\mathrm{C}(0.4 \mathrm{mg} / \mathrm{mL})$ and applied to the stenotic area in a radial fashion using rigid forceps. The amount of time the trachea was exposed to the topical mitomycin $C$ was about 15 seconds for each application. Clean gauze with fresh mitomycin $\mathrm{C}$ was used for each application on the trachea.

\section{CASES}

Case 1: The patient is a 45-year-old woman with type II diabetes, hypertension, dyslipidemia, and interstitial lung disease who presented to the emergency room complaining of her "throat closing up" as well as shortness of breath. This patient was previously intubated for 14 days in Mexico secondary to respiratory failure from a lung infection. Prior to her admission at our hospital she was admitted for shortness of breath twice after her initial intubation. A CT scan of her neck on admission revealed smooth narrowing of the trachea at the level of C7 through T1, measuring approximately $3 \mathrm{~mm}$ in transverse, $5 \mathrm{~mm}$ in A-P, and $8 \mathrm{~mm}$ in craniocaudal dimensions. Bronchoscopy revealed tracheal stenosis $7 \mathrm{~cm}$ from the vocal cords with greater than $50 \%$ narrowing secondary to granulation tissue. Rigid bronchoscopy was used to perform balloon dilation, EES, and ablation of granulation tissue via APC. The stenotic area was dilated to $75 \%$ of her native trachea. Topical mitomycin $C$ was then applied at the site of granulation tissue. Repeat bronchoscopy approximately one month later revealed no significant tracheal stenosis. After presenting to the hospital several months later with a respiratory infection, the patient was found to have a $30 \%$ obstructing lesion in her mid trachea secondary to tracheal stenosis. This was associated with dynamic collapse of her mid trachea. This area was treated with cryotherapy and balloon dilation with no significant return of stenosis or dyspnea.

Case 2: The patient is a 54-year-old woman with asthma, congestive heart failure, mitral stenosis, and hypertension who was admitted to the medical intensive care unit (MICU) and intubated secondary to respiratory failure from an acute asthma exacerbation and Influenza A infection. The patient was intubated for three days. The patient developed stridor and shortness of breath approximately four weeks after discharge. Direct laryngoscopy performed at this time ruled out laryngeal stenosis. A CT scan of the neck did not reveal any abnormalities. A 3-D CT scan of the chest showed a short segment of narrowing within the trachea at the thoracic outlet. Flexible bronchoscopy and radial endobronchial ultrasound revealed a tracheal lumen less than $5 \mathrm{~mm}$ with circumferential granulation tissue approximately $5 \mathrm{~cm}$ below the vocal cords (Figure 1). Rigid bronchoscopy and balloon dilation were performed with subsequent cryotherapy of the stenotic area. This was followed by ablation of stenotic tissue with APC and topical application of mitomycin $\mathrm{C}$ at the site of granulation tissue. Postprocedure the tracheal diameter was $1.2 \mathrm{~cm}$ with a lumen size greater than $75 \%$ normal (Figure 2). At the patient's follow-up appointment approximately two weeks later she noted mild, occasional stridor but no significant dyspnea. Repeat bronchoscopy showed $10 \%$ occlusion in mid trachea secondary to granulation tissue (Figure 3). This was again treated with cryotherapy via rigid bronchoscopy, APC, and balloon dilation. The patient has remained asymptomatic for several months after her last procedure.

\section{Case 2 Images \\ Post-intubation tracheal stenosis}




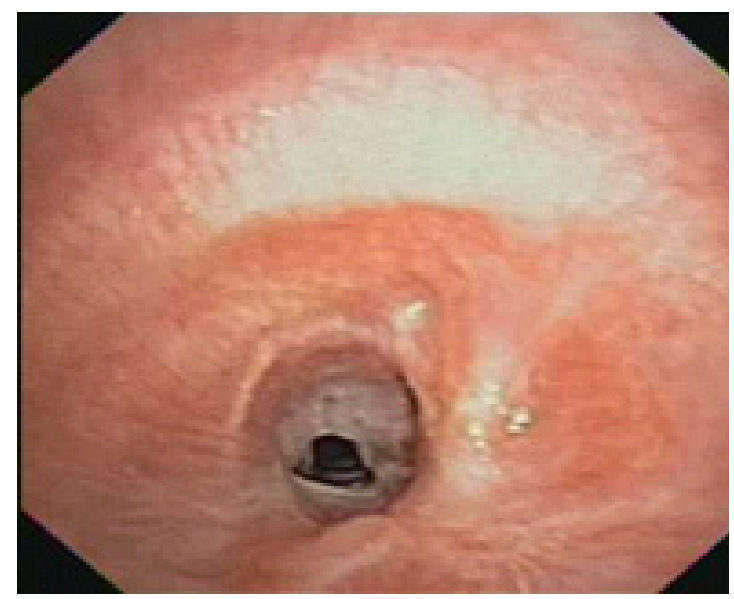

Figure 1: Bronchoscopic visualization of trachea revealing greater than $50 \%$ stenosis prior to intervention

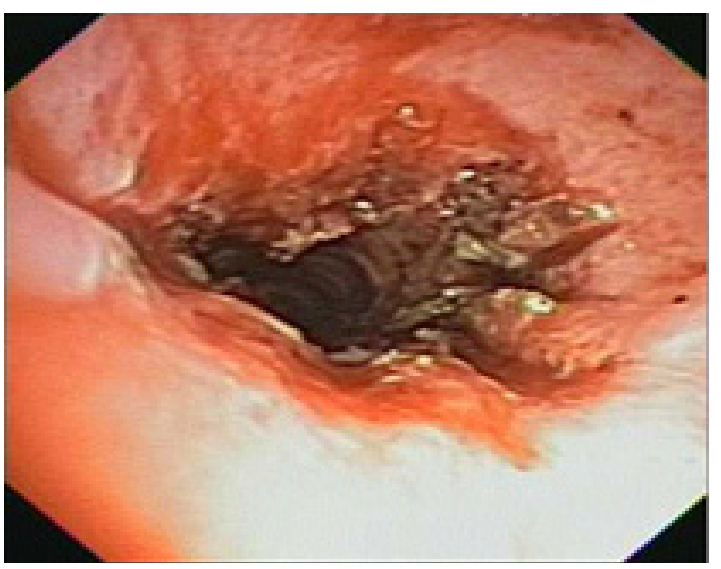

Figure 2: Post intervention and application of topical mitomycin C

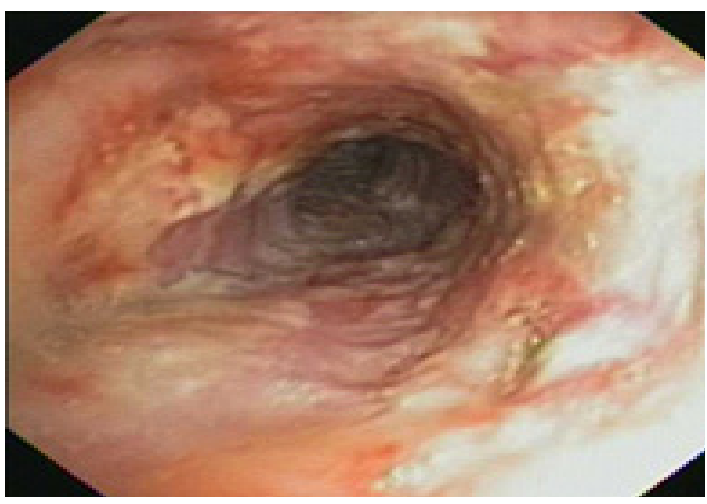

Figure 3: Follow-up bronchoscopy approximately one week after intervention revealing normal tracheobronchial tree
Case 3: This patient is a 39-year-old woman with a cardiomyopathy status-post AICD, type II diabetes, and chronic respiratory failure. The patient had open tracheostomy after prolonged mechanical ventilation for acute ischemic stroke. Approximately seven weeks later she developed acute respiratory distress associated with increasing peak pressures and difficulty with passing the suction catheter. Bronchoscopy showed $90 \%$ narrowing of the trachea secondary to granulation tissue (Figure 4). The granulation tissue was debrided using cryotherapy, and then the patient underwent balloon dilation (Figure 5). Repeat bronchoscopy three weeks later showed recurrence of granulation tissue which was again treated with cryotherapy and balloon dilatation (Figure 6). Topical mitomycin $\mathrm{C}$ was applied at the site of granulation tissue on the third bronchoscopic evaluation with a resulting tracheal diameter greater than $75 \%$ normal (Figure 7). The patient presented to the emergency room four weeks later with acute dyspnea secondary to recurrence of tracheal stenosis and died before any intervention could be performed.

\section{Case 3 Images Post-tracheostomy tracheal stenosis}

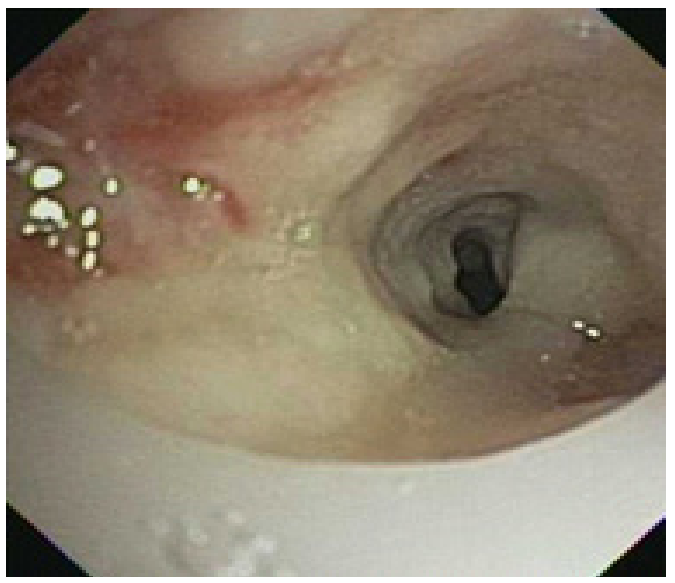

Figure 4: Initial bronchoscopy showing greater than $90 \%$ narrowing of trachea 


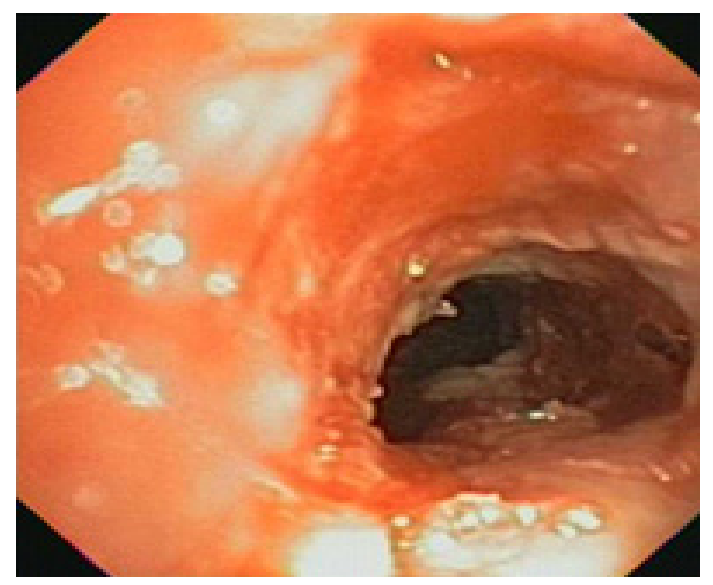

Figure 5: Post intervention with cryotherapy and CRE balloon dilation

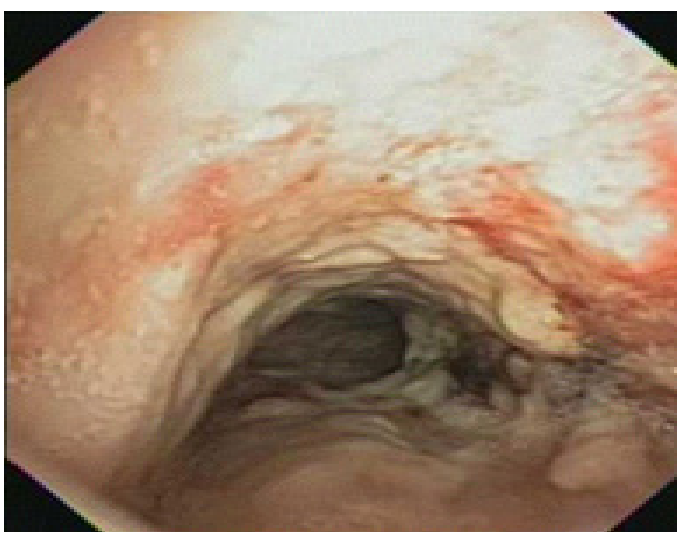

Figure 6: Repeat bronchoscopy three weeks after initial intervention showing recurrence of granulation tissue

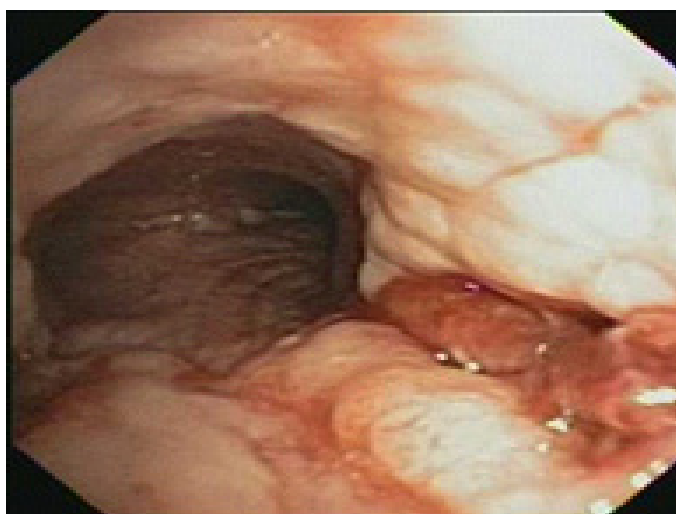

Figure 7: Post intervention and application of topical mitomycin C
Case 4: The patient is a 47-year-old woman with a seizure disorder and depression who was intubated for over two weeks for acute respiratory failure after attempting suicide by hanging. The patient was admitted to the MICU one month later and intubated for worsening shortness of breath, non-productive cough, and hoarseness. A CT scan of the chest on admission showed tracheal stenosis, and the patient underwent endoscopic balloon dilation for tracheal stenosis. The patient was eventually extubated and discharged home with no symptoms of dyspnea or stridor. She was readmitted one month later with a mild exacerbation of her stridor partially relieved by oral corticosteroids. Rigid bronchoscopy and balloon dilation were performed with subsequent cryotherapy of the stenotic area. This was followed by ablation of stenotic tissue with APC, and topical mitomycin C application at the site of granulation tissue. Approximately one month later the patient was again treated for symptomatic tracheal stenosis. Upon visualization with rigid bronchoscopy the patient was found to have greater than $75 \%$ stenosis in the upper trachea. This was treated with balloon dilation with no significant change in the airway diameter. The patient's oxygen saturation remained suboptimal, and the patient had an emergent tracheostomy at this time.

\section{Discussion}

Tracheal stenosis is a rare and serious complication after intubation. Common treatments for this complication include Nd: YAG laser, EES, APC, electrocautery, balloon dilation, stenting, cryotherapy, and surgical intervention. ${ }^{1}$ Surgery is currently the definitive treatment but carries a significantly higher risk for adverse events as it often involves complex procedures. Application of topical mitomycin $\mathrm{C}$ has recently been described as a potential addition to the treatment of tracheal stenosis. ${ }^{2}$ This method for the prevention and treatment of tracheal stenosis has been successful in studies involving animal models. ${ }^{3}$ Limiting the duration of intubation time is crucial in preventing complications associated with intubation. However, patients requiring tracheostomy may have more severe recurrent tracheal stenosis, leading to increased complications, if they develop tracheal stenosis after this procedure. This may represent a dif- 
ferent pathologic process and need different evaluation and management. Our findings related to this subgroup are similar to those reported by Zias, et al. ${ }^{4}$

\section{Conclusion}

The topical application of mitomycin $\mathrm{C}$ following endoscopic electrosurgery can be used for treatment of post intubation tracheal stenosis. Our experience with these four cases shows a good initial success rate with topical mitomycin C application. Most patients required a repeat intervention a few weeks later. The success with topical mitomycin C seems more likely in patients with post-intubation than with post-tracheostomy tracheal stenosis. Our results with bronchoscopic therapy and topical application of mitomycin $C$ suggest that this intervention works better as a bridge to definitive surgery rather than as a stand-alone therapy.

Author Affiliation: All authors work in the Department of Internal Medicine at Texas Tech University Health Sciences Center in Lubbock, TX. Audra Fuller is a resident, Mark Sigler is a fellow in pulmonary and critical care medicine, and Raed Alalawi is a faculty member in the Division of Pulmonary and Critical Care Medicine. Shrinivas Kambali was a fellow in pulmonary and critical care medicine.

Received: $12 / 22 / 2014$

Accepted: $1 / 8 / 2015$

Reviewers: Kenneth Nugent MD

Published electronically: 01/15/2015

Conflict of Interest Disclosures: None

\section{REFERENCES}

1. Cavaliere S, Foccoli P, Toninelli C, Venuta F, La Face B. Endoscopic treatment of malignant airway obstructions in 2,008 patients. Chest 1996; 110:1536-542.

2. Simpson, Blake C, James J. The efficacy of mitomycin$\mathrm{c}$ in the treatment of laryngotracheal stenosis. The Laryngoscope 2006; 116:1923-925.

3. Eliashar R, Eliachar I, Esclamado R, Gramlich T, Strome M. Can topical mitomycin prevent laryngotracheal stenosis? The Laryngoscope 1999; 109:594-600.

4. Zias N, Chroneou A, Tabba M, Gonzalez A, Gray A, Lamb C, Riker D, Beamis J. Post tracheostomy and post intubation tracheal stenosis: report of 31 cases and review of the literature. BMC Pulmonary Medicine 2008; 8:18. 